marquée, avec un sillon fin peu visible en forme d'accent circonflexe. - Épistome situé dans un plan différent de celui du front et transversalement concave. - Prothorax lisse, très luisant, très bombé, strie marginale entière forte, angles antérieurs arrondis à peine marqués, postérieurs légèrement obtus, base avec une fine bande de petits points serrés, non cilié sur les còtés. - Écusson triangulaire à peine visible. - Elytres à stries obsolètes presque invisibles, la première dorsale seule bien marquée dans sa partie postérieure, presque entière, sinueuse, les autres indiquées seulement par une fine ligne de points pas plus forts que ceux de la ponctuation, 2-3 raccourcies au milieu, 4 réunie à la suturale par un arc basal et raccourcie des la base. Suturale entière se prolongeant le long du bord apical et allant rejoindre la marginale. Subhumérale externe nulle, interne bien marquée, courte, réunie à l'humérale qui est peu visible. Toute la surface ponctuée sauf le bord latéral et l'épaule, ponctuation pas très dense formée de gros points en forme de cicatrices. - Pygidium très grand, bombé, finement et densément ponctué ainsi que le propygidium. - Prosternum en carène tranchante, très concave à la partie antérieure, stries réunies presque dès la base limitant un triangle lisse à sommet très aigu. - Mésosternum peu visiblement ponctué, bordé d'une fine strie entière. - Jambes antérieures fortement bidentées. Cuisses postérieures en ovale aplati à tranche aiguë, très bombées, lisses, brillantes, fortement ciliées en dessous. Tibias postérieurs analogues à ceux du Pachylopus Henoni Schmidt, mais moins dilatés et moins épais, à épines de la marge externe plus courtes, plus robustes, a tarses moins longuement ciliés. Long. 2,7 mill.

Se distingue de P. Henoni Schmidt par sa taille plus petite, son pronotum lisse à la partie antérieure, ses stries obsolètes et ses élytres ponctués sur tout le disque.

Je dédie cette nouvelle espèce de Pachylopus à M. Joh. Schmidt qui a décrit la première du genre trouvée en Algérie.

Un exemplaire de ma collection et un de la collection Joh. Schmidt, provenant des chasses d'A. Hénon à Aïn-Sefra (province d'Oran).

\title{
Description de six Coléoptères de l'île Ishigaki-Sima
}

Par L. Fairmaire.

Notre collègue, M. R. Oberthür, ayant reçu quelques insectes recueillis dans l'île d'Ishigaki-Sima, située au nord-est de Formose, et inexplorée jusqu'à présent, a bien voulu les partager avec moi. Presque toutes les espèces sont nouvelles; la plus intéressante est peut-être un Pachy- 
rhynchus noir qui est, à ce que je crois, le plus septentrional du genre. On n'en a pas signalé au Japon, peut-être en trouvera-t-on à Formose.

Outre les six insectes décrits ci-après il y a encore un Eucyrtus multicolor Fairm., décrit de Bornéo, et l'Alphitobius nitidulus Motsch., qui se trouve depuis Ceylan jusqu'aux iles Andaman et à Java.

Onthophagus lutosopictus n. sp. - Subovatus, modice convexus, elytris planiusculis, fusco-aenescens, vix nitidulus, lateribus breviter fulvo-hirtulus, elytris basi utrinque maculis 3 sat minutis, lutosis, apice parum regulariter lutoso maculosis et lateribus interdum lituris oblongis similibus ornatis, pygidio utrinque lutoso maculato; capite dense transversim strigosulo, margine antico reflexo, medio truncatulo, utrinque rotundato, medio carinula transversa paulo arcuata, utrinque abbreviata, vertice summo transversim acute carinato, antennis rufopiceis, clava fusca; prothorace transverso, elytris medio haud angustiore, antice et basi aequaliter angustato, lateribus et angulis posticis rotundatis, dorso dense sat subtiliter punctato-rugoso, antice medio brevissime obtuso-lobato; elytris subtiliter striatis, intervallis laxe asperatis, basi planatis, apice paulo convexis, sutura apice elevata; pygidio asperopunctato; subtus cum pedibus magis fuscus et nitidus, tibiis anticis intus pilosis, extus dentibus 4 sat latis obtusis armatis. - Long. 8 mill.

Les ơ ont les tibias antérieurs plus longs, la carène clypéale plus relevée au milieu.

Voisin du ceylonicus Har. pour la forme de la tête et le dessin des élytres, mais bien distinct par sa teinte mate et les taches bien séparées de la base et de l'extrémité des élytres; plus voisin, seulement sous ce dernier rapport, de l'o. gonoderus Fairm., du Yunnan.

Eurylampyris bivitrea n. sp. - Oblonga, parum convexa, rufa, opaca, elytris nigris, opacis, capite, palpis antennisque nigris; oculis mediocribus, sat distantibus, antennis compressis, valde serratis, corporis medium vix attingentibus, apice minus latis, articulo ultimo oblongo, palpis maxillaribus articulo ultimo acuminato, prothorace parum transverso, elytris paulo angustiore, a basi antice angustato, lateribus cum margine antice rotundatis, dorso medio convexo, ad latera late deplanato, margine antico praecipue reflexo, antice plagulis 2 translucidis bene determinatis, oblongis, disco medio obsolete longitudinaliter carinulato et utrinque obsolete impresso, margine postico recto, angulis sat obtusis, dorso linea obsolete elevata, antice spatiis 2 vitreis, bene limitatis, insignito; scutello rufo, oblongo, attenuato, convexo; elytris sat amplis, ad humeros late rotundatis, apice rotundatis, dorso dense subtiliter coriaceis et utrinque nervulo parum distincto signatis; segmentis ven- 
tralibus ultimis lateribus angulatim dilatatis, pedibus nigris, compressis, late leviter canaliculatis. - Long. 12 - 17 mill.

Ressemble ì l'E. luteicollis Kraatz, mais plus grand, plus roux, avec le corselet moins court, presque anguleusement arrondi en avant, le bord antérieur bien plus mince, les deux taches plus nettes, plus hyalines, le disque convexe, très finement caréné et les élytres à nervures fines mais distinctes.

Amarygmus callichromus n. sp. - Forme et coloration de l'iridipennis Fairm., de Sumatra; seulement le corselet ne présente qu'une large bande cuivreuse au milieu tandis qu'il y en a deux étroites chez l'espèce de Sumatra; les élytres sont un peu plus fortement ponctués dans les stries et sur les intervalles; la partie prosternale a, de chaque côté, un profond sillon qui est à peine indiqué dans l'autre espèce, le $4^{\text {e }}$ segment de l'abdomen est notablement plus court que le précédent et les épipleures sont d'un vert bronzé métallique et non d'un brun foncé. - Long. 10-11 mill.

Pachyrhynchus infernalis n. sp. - Ovato-oblongus, valde convexus, bipartitus, totus niger, nitidus; capite rostroque politis, hoc inter antennas fortiter transversim profunde impresso, apice latiore, punctulato, fronte inter oculos lineola transversim impressa; prothorace globoso, laevi, antice et basi aequaliter angustato, lateribus basi breviter rectis, dorso laevi, basi medio linea impressa mox evanescente signato; elytris brevissime ovatis, valde convexis, subgibbosis, postice magis angustatis, dorso laevibus, costulis obsolete elevatis plus minusve distinctis et vage lineatim laxe punctulatis; subtus subtilissime vix distincte roriaceus, pedibus magnis, femoribus clavatis, tibiis compressis, anticis leviter arcuatis, tarsis latis. - Long. 11 mill.

Forme et taille du $P$. moniliferus, mais entièrement noir, le front plus fortement creusé, sans sillon médian, ni ponctuation, le corselet n'ayant pas de sillon médian, mais une impression basilaire peu profonde; les élytres montrent aussi quelques còtes à peine indiquées, plus marquées sur les còtés, ayant quelques points en lignes; la suture entre les $1^{\text {er }}$ et $2^{\text {e }}$ segments ventraux est à peine distincte, mais présente trois impressions.

Camptorhinus squamosohirtus n. sp. - Oblongus, haud parallelus, convexus, fuscus, indumento lutoso-cervino vestitus, griseo et fusco variegatus; capite squamulis hirsuto, inter oculos bifasciculato, rostro gracili, leviter arcuato, polito, basi medio carinulato, lateribus rugosulo; prothorace elytris angustiore, longitudine vix latiore, antice paulo, 
postice cix angustato, dorso dense fere rugose punctato, utringue vitta pallidiore antice cum altera conjuncta, squamis erectis sparsuto, diseo penicillis 2 fuscis signato; elytris oblongis, ad humeros angulatis, medio leviter ampliatis, post medium angustatis, dorso striatis, striis lace punctatis, intercallis fereplanis, medio fascia angulari communi albida, antice et postice fusco marginata, lituris aliquot fuscis et albidis sparsuto et sefulis clacatis parce hirto; femoribus clacatis, subtus dente triangulari acuto armatis, cum tibîis fusco annulatis, - Long. 5 mill. (rostr. excl.).

S'éloigne de la majorité des Camptorhinus par sa forme non parallêle et se rapproche au contraire d'une espêce de Madagascar, le C. denticaudatus Fairm.

\section{Gen. Pachyosa n. gen.}

Genre voisin des Merosa dont il differe par les antennes plus épaisses, garnies en dessous d'une villosité serrée, le $3^{e}$ article à peine plus long que le $4^{\ominus}$, le corselet à côtés parallèles, non tuberculés, rebordé à la base. La tête est un peu creusée entre les antennes, les yeux sont complètement divisés, le labre est três grand, saillant, débordé par les mandibules, qui sont grandes et nobustes, le chapenon bondé en avant par une ligne faiblement saillante, transversale maís légèement arquée; le métasternum est soudé avee le mésosternum qui est obtusément saillant et faiblement échaneré á l'estrémité; le 1er artiele des tarses est à peine plus long que le 28 .

Pachyosa cervinopicta n. Sp. - Oblonga, concé̉er, nägra, opaca,

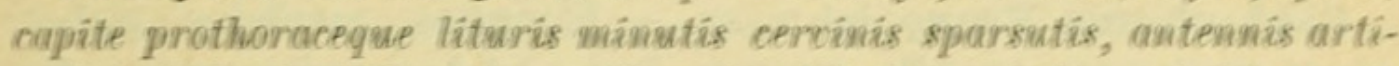
calis $3-11$ basi albido pubescentíbus, 2 ultimais brecissime, elytrís cercino-trifasciolatis, fasciüs valde dilaceratis, $1^{\mathrm{a}}$ basală, $2^{\mathrm{a}}$ mediana, $3^{\mathrm{a}}$ anteapicali et margîne apicalì símîliter anguste colorata; fronte quadrata, medio sulcata, clypeo antice et labno sat longe dense cercino pílosís, mandituläs metallice natidis; antennis corpore haud longioribus, apicem versus gracilia ribus, articulo ultimo brecione; prothonace trasevensim guadrato, capite hawd latiore, elytris angustiore, lateribus fere rectis,

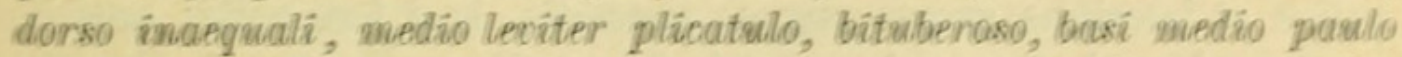
tubenaso, et fisso, antice trasscersim âmpresso; elytrís basi trumcatis, ad humenos angulatis, dorso pubescentäbus, parum regulariter asperulo-punctatis, basi fortìus et paulo granulatís; subtucs cum pedibus cernäno fasciolata, abdománis segmentís apice anguste cerníno marginatis, - Long. 15 mill.

Les tubereules du eorselet sont un peu variables; les deux discoj- 
daux sont parfois peu marqués, le postérieur est tantôt bien saillant, entier, tantòt assez faible et sillonné.

\section{Description de Malacodermes nouveaux recueillis en Océanie par $\mathbf{M}$. Ie $\mathbf{D}^{\mathrm{r}} \mathbf{P h}$. François [CoL.]}

Par J. Bourgeors.

Cladophorus gagates n. sp. - Elongatus, parallellus, glaber, aterrimus; capite prothoraceque nitidiusculis, illo fossula profunda, elliptica, spatium interoculare omnino occupante, transversim excavato, hoc longitudine basi fere aequali, antice valde rotundato, lateribus subparallelis, medio paululum coarctatis, angulis anticis subrotundatis, posticis fere rectis, disco כ̈-areolato (areola mediana inaequaliter bilanceolata), in triente anteriori carinato, margine antico punctis grossis fortiter impresso, basi utrinque leviter sinuata; scutello postice rotundatim emarginato; elytris 9-costatis, costis alternis multo elevatioribus, interstitiis crebre quadrato-areolatis; corpore subtus pedibusque nitidiusculis.

0 . Oculis magnis, prominentibus; antennis a $3^{\circ}$ articulo inde longe flabellatis; abdominis segmento penultimo postice rotundatim profunde emarginato.

ค. Hucusque invisa.

Long. 7, , mill. ; lat. 2 mill.

Vanikoro (Dr. Ph. Francois). Un ex.

Cladophorus Françoisi n. sp. - Elongatus, parallelus, glaber, niger, prothorace, scutello, angusto limbo ad basim elytrorum parteque anteriori suturae rufo-testaceis; fronte deplanata, rufescente; prothorace nitidiusculo, subquadrato, longitudine basi aequali, antice rotundato, lateribus fere parallelis, subrectis, angulis anticis rotundatis, posticis fere rectis, disco areola bilanceolata, sat angusta, antice in carinam evadente, postice aperta, medio longitudinaliter exarato, spatio laterali utrinque a plico transverso obsolete bipartito, margine antice et lateribus grosse rugoso-punctatis, basi fere recta; scutello postice triangulariter exciso; elytris 9-costatis, costis alternis eleratioribus, insterstitiis crebre quadrato-areolatis; corpore subtus pedibusque nitidiusculis, mesosterno, coxis, trochanteribus femorumque basi rufo-testaceis.

o. Antennis a $3^{\circ}$ articulo inde longe acutissimeque serratis; abdominis segmento penultimo profundissime triangulariter exciso, fere bipartito, ultimo elongato-triangulari. 


\section{$2 \mathrm{BHL}$ Biodiversity Heritage Library}

Fairmaire, Léon. 1897. "Description de six coléoptères de l'Îsle Ishigaki-Sima." Bulletin de la Société entomologique de France 1897, 68-72.

https://doi.org/10.5962/bhl.part.29501.

View This Item Online: https://www.biodiversitylibrary.org/item/36398

DOI: https://doi.org/10.5962/bhl.part.29501

Permalink: https://www.biodiversitylibrary.org/partpdf/29501

\section{Holding Institution}

Smithsonian Libraries

\section{Sponsored by}

Smithsonian

\section{Copyright \& Reuse}

Copyright Status: NOT_IN_COPYRIGHT

This document was created from content at the Biodiversity Heritage Library, the world's largest open access digital library for biodiversity literature and archives. Visit BHL at https://www.biodiversitylibrary.org. 